\title{
A Novel Energy Efficient Clustering Algorithm for Wireless Sensor Networks
}

\author{
Santar Pal Singh \\ Electronics and Computer Discipline, DPT \\ Indian Institute of Technology Roorkee \\ Roorkee, India \\ spsingh78@gmail.com
}

\author{
Subhash Chander Sharma \\ Electronics and Computer Discipline, DPT \\ Indian Institute of Technology Roorkee \\ Roorkee, India \\ scs60fpt@iitr.ac.in
}

\begin{abstract}
Wireless sensor networks (WSNs) are composed of huge amount of tiny resource constrained devices known as sensors. The demand for an energy efficient structure is becoming gradually more essential in WSNs. Energy efficient clustering is a distinguished optimization problem which has been studied extensively to expand the life span of WSNs. In this manuscript, a new energy efficient clustering scheme for WSNs with the use of Particle Swarm Optimization (PSO) is proposed. The clustering algorithm is implemented with the goal of concurrently minimizing the intra-cluster distance along with optimizing the usage of network energy. The presented algorithm is tested widely and results are analyzed and compared with previous ones to show the supremacy in term of alive nodes, energy consumption, packet delivery ratio, and system throughput. Simulation results show that the proposed algorithm outperforms other existing algorithms.
\end{abstract}

Keywords-wireless sensor networks; clustering algorithms; energy efficiency;particle swarm optimization

\section{INTRODUCTION}

Latest advancements in wireless and embedded technologies allow the use of micro autonomous system comprised of small tiny devices known as sensors. These sensors can detect, compute and communicate via suitable sensor technology which results to a wireless sensor network. Deployment ease and low cost sensors make wireless sensor network suitable for many applications like health care, transportation, smart building, and environmental monitoring etc. Regarding the WSN design, there are lots of significant aspects that ought to be considered such as the size of nodes, hardware complexity and low energy utilization $[1,2]$. Energy efficiency is considered to be the main design objective, in view of the fact that a wireless node can only be outfitted with a restricted power supply. In several applications, replacement of energy sources is not possible, and thus the node's life span demonstrates a strong reliance on battery life $[2,3]$.

Clustering is among the design schemes utilized to handle the energy utilization proficiently, via minimizing the amount of nodes to be included in long-haul transmission with sink and allocating the energy expenditure uniformly amid the nodes. In this scheme, every set of nodes has a head node recognized as Cluster Head $(\mathrm{CH})$ that performs data aggregation on data as of its relevant group and forwards it to sink or base station (BS). Thus, the clustered schemes have the advantages of decreasing the total information that requires to be disseminated, besides improving resource allocation as well as bandwidth reusability [4-7].

Lots of algorithms have been reported with the goal of exploiting the network life span by implementing clustering designs [6-10]. Probably the most renowned hierarchical protocol is Low Energy Adaptive Hierarchy (LEACH). LEACH is a hierarchical scheme that introduces disseminated cluster establishment; at some stage in it, nodes choose themselves as head node with some possibility [11]. This algorithm is executed sporadically and the possibility of becoming $\mathrm{CH}$ for every epoch is elected to insure that each node turn out to be a $\mathrm{CH}$ at least on one occasion in $1 / \mathrm{p}$ rounds, wherever $\mathrm{p}$ is preset proportion of CHs. It gives considerable energy saving and lengthened network life span over traditional routing methods for example minimum transmission energy (MTE) scheme. However, LEACH does not ensure that preferred amount of $\mathrm{CHs}$ is elected and $\mathrm{CHs}$ are not uniformly placed in the network. The most important drawback of LEACH is that a sensor node with incredibly low energy might be designated as $\mathrm{CH}$ and the $\mathrm{CHs}$ send the data to base station in one-hop manner [11]. Thus, this scheme increases the energy utilization of the CHs. Various methods have been designed to improve LEACH i.e. LEACH-C, PEGASIS, HEED etc. [1214]. LEACH-C behaves superiorly compared to LEACH because of the appropriate election of $\mathrm{CH}$ by $\mathrm{BS}$. In the selection of $\mathrm{CH}$, it considers the energy as well as distance into account [12]. Hence, LEACH-C can efficiently choose the $\mathrm{CH}$ by taking into consideration of energy competence and be capable of extending the network life span. PEGASIS arranges the nodes into chain with the intention that every node sends and/or receives the data merely as of its nearby nodes. In every round an arbitrarily chosen node from chain is elected as cluster head. PEGASIS improves the network lifetime 
compared to LEACH but data delay is considerably high [13]. So, it is not suitable for large area networks. The HEED periodically chooses the CHs on the basis of node's remaining energy along with connectivity measure of nearest node or node degree [14]. Other LEACH based clustering algorithms have been presented [15-18].

In the present paper, a novel energy efficient clustering algorithm for WSNs with help of the PSO algorithm is investigated aiming to maximize the life span of network. The key idea of the proposed algorithm is the election of a $\mathrm{CH}$ that focuses on minimization of intra-cluster distance and optimization of network energy. The proposed method uses a high energy node as $\mathrm{CH}$ and makes clusters which can be uniformly placed in the sensor region. The use of PSO has been suggested earlier in $[19,20]$. In the current approach, we attempted to optimize the energy usage of nodes whereas maximizing the data communication. The main difference among this work and earlier works is the use of PSO to decide the optimum nodes as $\mathrm{CHs}$ to expand the life span of the system

\section{SYSTEM MODEL}

Here, we illustrate the network model and energy model used in the proposed algorithm.

\section{A. Network model}

Let us consider a net model alike to that utilized in LEACH with the following characteristics:

- Every node carry out sensing tasks sporadically and all the time send data to BS.

- A fixed BS can be placed inside or outside the WSN area.

- Each and every node is fixed and energy restrained.

- Each and every node is capable of working in $\mathrm{CH}$ mode as well as in sensing manner.

- Fusion of data is utilized to lessen the overall data.

\section{B. Radio energy model}

In this work, we employ energy model of radio (REM) as given in Figure 1.

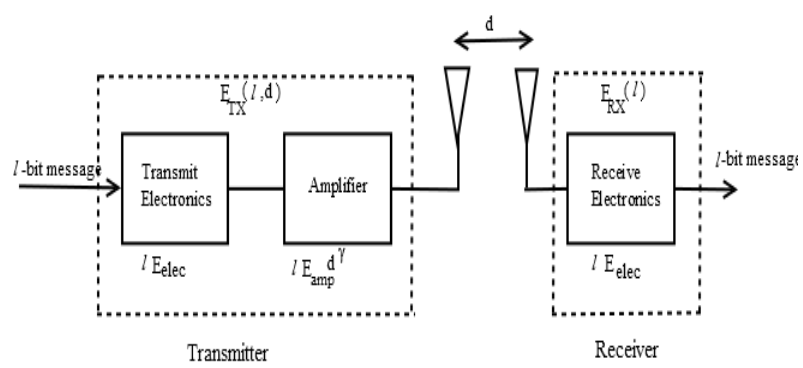

Fig. 1. Radio energy model

In REM, transmitter depletes energy to operate radio as well as power amplifier, moreover the receiver uses up the energy to operate radio $[21,22,25]$. The radios can carry out the power control and thus utilize minimum energy needed to get to the destined recipients. Because of attenuation, energy loss model is applied for small and long distances. Hence, to realize signal-to-noise (SNR) ration during broadcasting $l$-bit packet over distance $d$, then energy dissipated in radio is specified as:

$$
E_{T X}(l, d)= \begin{cases}l E_{\text {elec }}+l \varepsilon_{f s} d^{2}, & \text { if } d \leq d_{0} \\ l E_{\text {elec }}+l \varepsilon_{m p} d^{4}, & \text { if } d \geq d_{0}\end{cases}
$$

Where $E_{\text {elec }}$ represents energy depleted per bit to operate the electronic circuitry, $\varepsilon_{f s}$ and $\varepsilon_{m p}$ depends on amp model, $d$ denotes the distance among sender and receiver, and $d_{0}$ denotes threshold transmission distance.

The threshold distance $d_{0}$ is given by:

Equating the equation (1) for $d=d_{0}$

$$
\begin{gathered}
l E_{\text {elec }}+l \varepsilon_{f s} d_{0}^{2}=l E_{\text {elec }}+l \varepsilon_{m p} d_{0}^{4} \\
d_{0}=\sqrt{\frac{\varepsilon_{f s}}{\varepsilon_{m p}}}
\end{gathered}
$$

To obtain $l$-bit packet, radio uses

$$
E_{R X}(l)=l . E_{\text {elec }}
$$

\section{PARTICLE SWARM OPTIMIZATION}

PSO is a swarm intelligence and collective intelligence based scheme, modeled behind the social foraging behavior of some animals like flock of birds or schools of fishes [23, 24]. In PSO, a swarm specifies the number of probable solutions to a complex problem, where each probable solution is known as a particle. The key objective of this method to discover the particle location that outcomes the finest assessment of a specified fitness function. During the initialization phase of swarm optimization, every particle set opening parameters in random fashion and is flown throughout the multidimensional search space. In every generation, every particle make use of the info about its earlier personal best position as well as global best position to maximize the possibility of moving in the direction of a better answer space that will results in improved fitness and update its candidate solutions in accordance with the subsequent equations:

$$
\begin{array}{r}
V_{i d}(t)=\omega \times V_{i d}(t-1)+c_{1} \times r_{1} \times\left(\text { Xpbest }_{i d}-X_{i d}(t-1)\right)+\ldots \\
\ldots+c_{2} \times r_{2} \times\left(\text { Xgbest }_{\text {. }}-X_{i d}(t-1)\right) \\
X_{i d}(t)=X_{i d}(t-1)+V_{i d}(t)
\end{array}
$$

Where $V$ and $X$ represents particle's velocity and place, $t$ is time, $c_{1}, c_{2}$ are learning coefficients, $\omega$ is the inertia parameter. $\mathrm{r} 1, \mathrm{r} 2$ denotes the numbers generated randomly among 0 and 1 . The Xpbest $_{i d}$ be the particle's best position individually and $X_{p b e s t}$ be the particle's best position globally. 


\section{PROPOSED ALGORITHM}

The flow sheet of proposed algorithm is revealed in Figure 2. The proposed algorithm is comprised of three core phases given as:

\section{Phase 1: Cluster formation}

The cluster is formed by the BS or sink node on the basis of centralized clustering. For clustering BS transmit a message to every node. The nodes after receiving this message starts to send its information like node ID, location, energy loss ratio, and current energy to sink. Based on such info, the BS estimates moderate energy level of every node. To assure that nodes that have acceptable energy level are likely to be a $\mathrm{CH}$ aspirant for present round. Later the sink uses the PSO scheme to identify the best $k \mathrm{CHs}$ which can minimize the cost function defined as:

$$
\text { Cost }=\alpha \times \text { func }_{1}+(1-\alpha) \times \text { func }_{2}
$$

Where func $_{1}$ denotes the maximum average distance of nodes to their corresponding $\mathrm{CHs}$ and can be expressed as:

$$
\text { func }_{1}=\max _{k=1 . . K}\left\{\sum_{\forall n_{i} \in C_{p, k}} \operatorname{dist}\left(n_{i}, C_{p, k}\right) /\left|C H_{p, k}\right|\right\}
$$

Where $\left|\mathrm{CH}_{p, k}\right|$ denotes the amount of nodes be owned by cluster $C_{k}$ for particle $p$. The func $_{2}$ represents the proportion of entire initial energy of the network with whole energy of $\mathrm{CH}$ candidates in present round. $\alpha$ is an user described constant utilized to weigh the effort of every sub-objective. The $f u n c_{2}$ can be expressed as:

$$
\text { func }_{2}=\frac{\sum_{i=1}^{N} E\left(n_{i}\right)}{\sum_{k=1}^{K} E\left(C H_{p, k}\right)}
$$

The fitness function has an aim of concurrently minimizing the intra-cluster distance among sensor nodes and relevant $\mathrm{CHs}$ and as well optimizing the network energy competence. In accordance with cost function, small rate of func $c_{1}$ and $f u n c_{2}$ recommend compact clusters through optimal set of nodes which have enough energy to execute the $\mathrm{CH}$ jobs.

For the WSN with $N$ number of nodes along with $K$ no. of clusters, the clustering schemes can be shown as:

Step1: Initializing particles to have $\mathrm{K}$ arbitrarily chosen $\mathrm{CHs}$ amid the entitled $\mathrm{CH}$ aspirants.

Step2: Estimate the function of cost of every particle:

(i) For every node $n_{i}, i=1,2, \ldots, N$

- Compute distance $\operatorname{dist}\left(n_{i}, \mathrm{CH}_{p, k}\right)$ among node $n_{i}$ and every heads $\mathrm{CH}_{p, k}$

- $\quad$ Allocate node $n_{i}$ to leader $\mathrm{CH}_{p, k}$, wherever

$$
\operatorname{dist}\left(n_{i}, C H_{p, k}\right)=\min _{\forall k=1 . . K}\left\{\operatorname{dist}\left(n_{i}, C H_{p, k}\right)\right\}
$$

(ii) Evaluate the cost function through Equation (5) to (7)
Step3: Discover the individual and overall best for every particle.

Step4: Updations of the velocity and position of particle with equation (3) and (4).

Step5: Updating the change in the particle's position assessment.

Step6: Depict the new updated location with nearby coordinates

Step7: Repeats steps 2 to 6 until the highest amount of iterations is achieved.

\section{Phase 2: Intra-cluster communication}

After clustering, routing procedure is invoked during data transmission stage. Routing consists of two steps: first one is route establishment and other one is forwarding sensed data. In route establishment phase route request message is broadcasted to all nodes with single hop transmission and unicast route reply message is sent in reverse path to source node. Once the route is established data transmission with multi-hop routing is commenced. In this work, the multi-hop communication protocol is utilized for data communication between nodes and $\mathrm{CHs}$ (intra-cluster communication) and $\mathrm{CHs}$ to $\mathrm{BS}$. Data aggregation is accomplished by the head node in each cluster for the intention of saving the remaining energy of nodes.

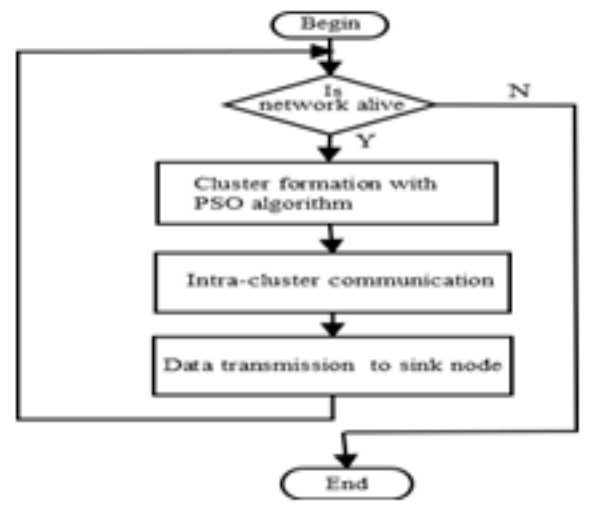

Fig. 2. Flow sheet of the proposed algorithm

\section{Phase 3: Data transmission to sink}

When the cluster heads have been chosen, the networks go into data communication phase. In this work, multi-hop communication protocol is utilized for communication among $\mathrm{CHs}$ and BS. The cluster head set up a threshold value i.e. d. In case of the transmission distance among $\mathrm{CH}$ and $\mathrm{BS}$ is lesser than the threshold value then the $\mathrm{CH}$ is committed to forward the calculated aggregated data to the head. Otherwise, $\mathrm{CH}$ will find next with minimum cost neighbor as a relay node. The minimum cost path and highest residual energy is calculated by using the formula given as:

$$
\operatorname{Cost}(j)=\gamma \times \frac{d\left(s_{i}, h_{j}\right)+d\left(h_{j}, B S\right)}{\max \max \left(d\left(s_{i}, h_{j}\right)+d\left(h_{j}, B S\right)\right)}+(1-\gamma) \times \frac{\max \max (E(j))-E(j)}{\max \max (E(j))}
$$


Where $\gamma$ is a randomized tuning parameter in the range $[0,1]$, $s_{i}, h_{j}$ are the sensor node and current head node respectively. Relay $\mathrm{CH}$ node is selected with minimum cost to send data to destination and inter-cluster routing is established when relay node is selected.

\section{RESULTS AND ANALYSIS}

The performance analysis of the proposed method is done with the help of NS-2.35. In the network, 100 nodes are organized in random fashion in $100 \mathrm{~m} \times 100 \mathrm{~m}$ area where BS is situated in network region. The performance assessment of proposed work is done as per the certain parameters given as:

- $\quad$ Energy consumption: total energy used by the entire nodes in their intra-cluster and inter- cluster activities.

- No. of alive nodes: quantity of nodes that have not so far exhausted their power.

- Packet delivery ratio: ratio of actual packet delivered to total packet sent.

- End to End delay: time utilized for message to be communicated transversely in network from source to destination.

- Throughput: quantity of packets per bytes received by source per unit time.

The proposed algorithm is evaluated with LEACH, LEACH-C, in term of alive nodes over rounds, packet delivery ratio, energy expenditure, end-to-end delay, throughput. The total rounds used in experiment are 500. The simulation parameters are summarized in Table I. In PSO scheme, we use 30 particles, $c_{1}=c_{2}=2.05, \omega=0.9$, and use this for 100 iterations and set $\alpha=0.5$ to provide equal involvement of every subobjective.

TABLE I. NETWORK PARAMETERS

\begin{tabular}{|c|c|}
\hline Parameter & Value \\
\hline Nodes & 100 \\
\hline Network size & $100 \mathrm{~m} \times 100 \mathrm{~m}$ \\
\hline BS position & $(50,100)$ \\
\hline Initial node energy & $2 \mathrm{~J}$ \\
\hline $\mathrm{E}_{\text {elec }}$ & $5 \mathrm{~nJ} / \mathrm{bit}$ \\
\hline $\mathrm{E}_{\mathrm{TX}}=\mathrm{E}_{\mathrm{RX}}$ & $50 \mathrm{~nJ} / \mathrm{bit}$ \\
\hline$\varepsilon_{\mathrm{fs}}$ & $10 \mathrm{pJ} / \mathrm{bits} / \mathrm{m}^{2}$ \\
\hline$\varepsilon_{\mathrm{mp}}$ & $0.0013 \mathrm{pJ} / \mathrm{bits} / \mathrm{m}^{4}$ \\
\hline $\mathrm{E}_{\mathrm{DA}}$ & $5 \mathrm{~nJ} / \mathrm{bit}$ \\
\hline Packet size & $500 \mathrm{bytes}$ \\
\hline No. of CHs & 5 \\
\hline No. of rounds & 500 \\
\hline &
\end{tabular}

Figure 3 shows the comparative analysis of energy consumption of the proposed algorithm with LEACH along with LEACH-C and it is clear that our algorithm have better energy usage in comparison to LEACH and LEACH-C. Figure 4 demonstrates the amount of nodes alive per round demonstrating stability of the system. Figures 5 and 6 illustrate the PDR and End-to-End delay over rounds in the network respectively. Figure 7 illustrates the throughput demonstrating the efficiency of the network.

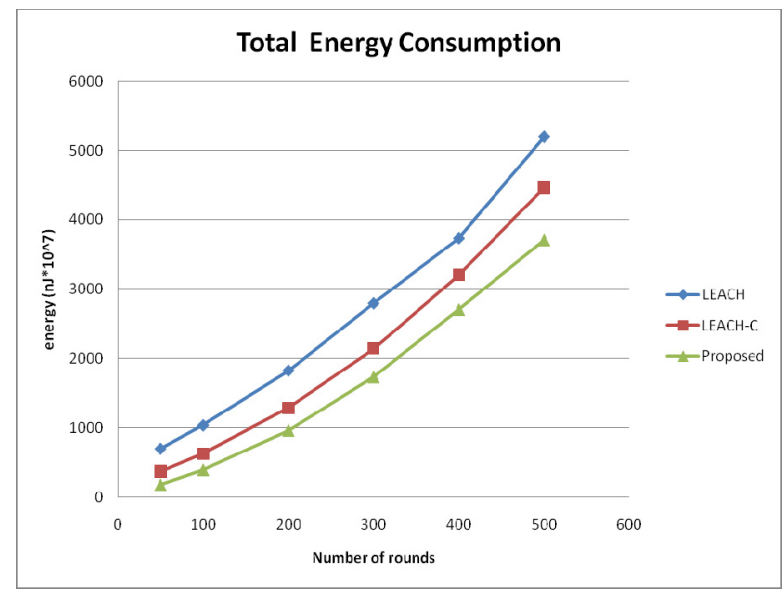

Fig. 3. Energy consumption over rounds

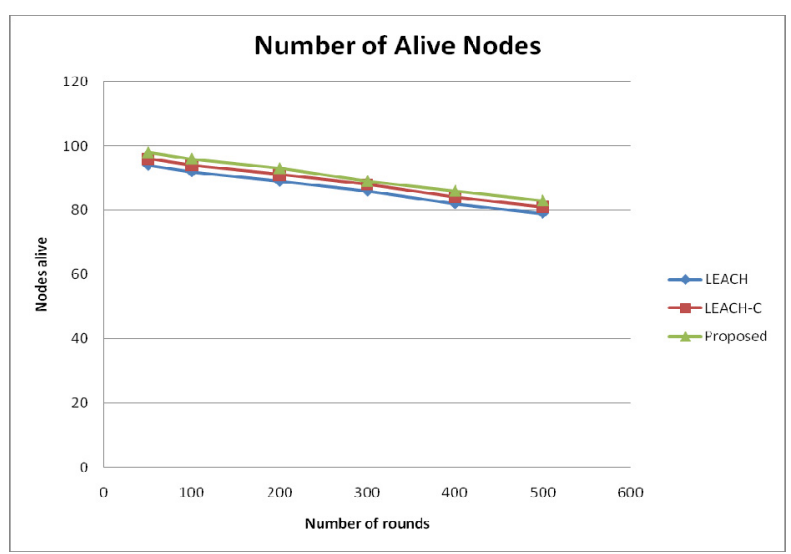

Fig. 4. Number of nodes alive over rounds

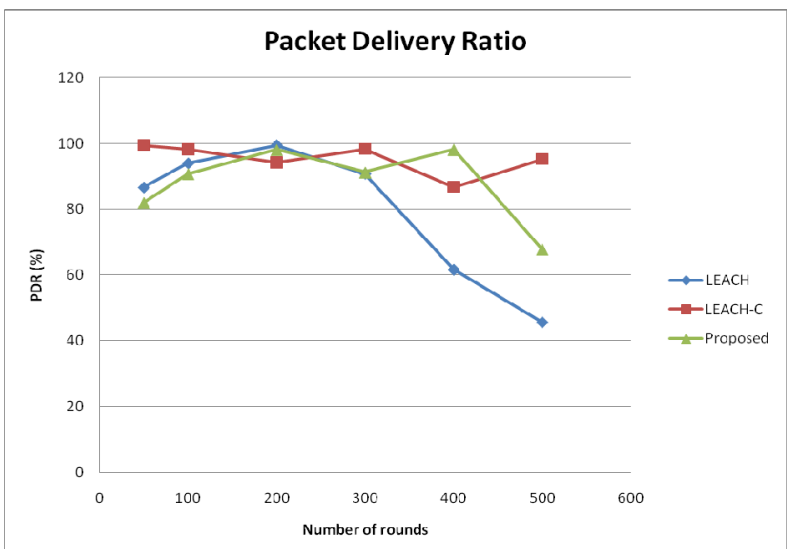

Fig. 5. Packet delivery ratio over rounds 


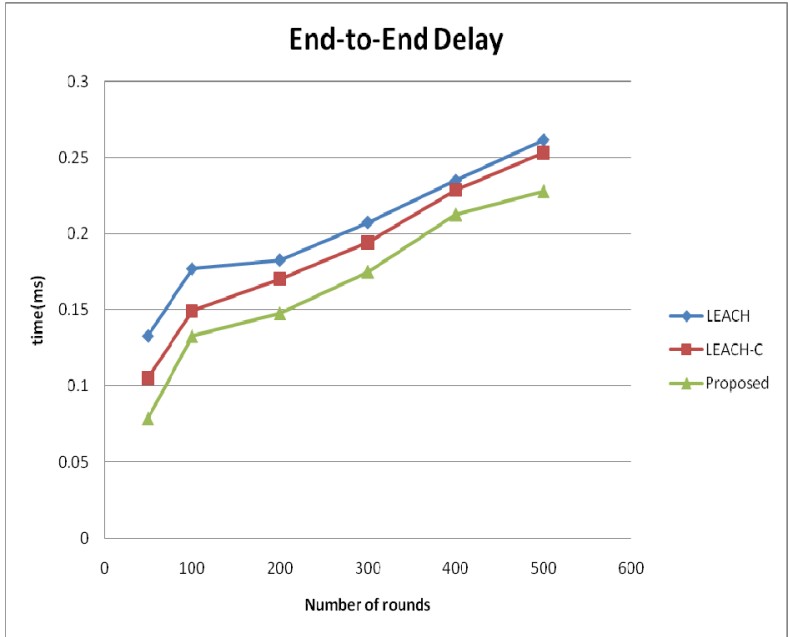

Fig. 7. End-to-End delay over rounds

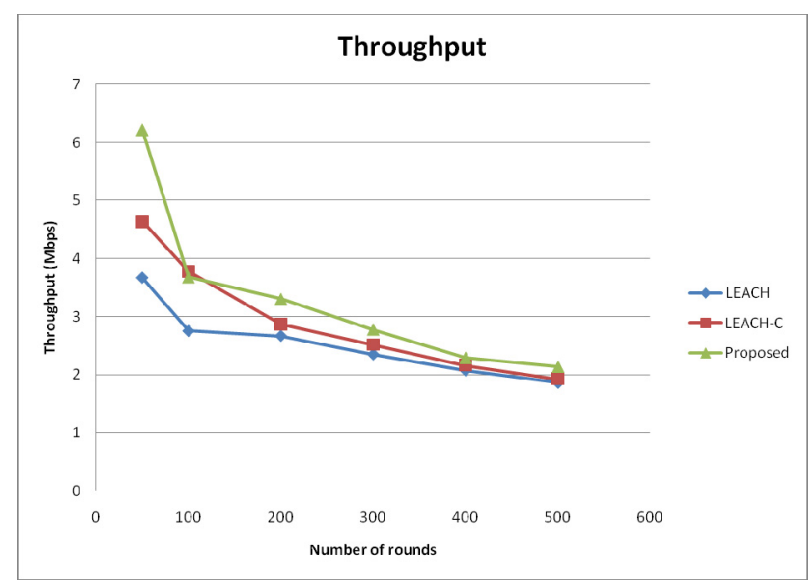

Fig. 8. Throughput over rounds

\section{CONCLUSION}

In this work, a novel clustering algorithm for WSNs with PSO method is presented. A new function of cost with the consideration of the maximum distance among a member node and their $\mathrm{CH}$ and the left over energy of $\mathrm{CH}$ aspirants in the $\mathrm{CH}$ election method is delineated. The results show that the proposed method offers better network stability in comparison to $\mathrm{LEACH}$ and LEACH-C. Moreover, the proposed algorithm induces better clustering via the uniform allocation of $\mathrm{CH}$ throughout the network. So, the proposed algorithm has improved energy efficiency and is useful in extending the network life span.

\section{ACKNOWLEDGMENT}

The authors like to thank MHRD (Govt. of India). This work is supported in part through a doctoral studies funding from ministry of human resource development.

\section{REFERENCES}

[1] I. F. Akyildiz, W. Su, Y. Sankarasubramaniam, E. Cyirci, "Wireless sensor networks: a survey", Computer Networks, Vol. 38, No. 4, pp. 393-422, 2002

[2] J. Yick, M. Biswanath, D. Ghosal, "Wireless sensor network survey", Computer Networks, Vol. 52, No. 12, pp. 2292-2330, 2008

[3] D. Gosain, I. Snigdh, "Performance comparison of protocols in bipartite wireless sensor networks", International Journal of Electrical and Computer Engineering, Vol. 5, No. 6, pp. 1417-1423, 2015

[4] A. A. Abbasi, M. Younis, "A survey on clustering algorithms for wireless sensor networks", Computer Communication, Vol. 30, No. 14, pp. 2826-2841, 2007

[5] X. Liu, "A survey on clustering routing protocols in wireless sensor networks", Sensors, Vol. 12, No. 8, pp. 11113-11153, 2012

[6] S. P. Singh, S. C. Sharma, "Cluster based routing algorithms for wireless sensor networks", International Journal of Engineering \& Technology Innovations, Vol. 1, No. 4, pp. 1-8, 2014

[7] S. P. Singh, K. Bhanot, S. Sharma, "Critical analysis of clustering algorithms for wireless sensor networks", Advances in Intelligent Systems and Computing, Vol. 436, pp. 783-793, 2015

[8] X. Liu, J. Shi, "Clustering routing algorithms in wireless sensor networks: an overview", KSII Transactions on Internet and Information Systems, Vol. 6, No. 7, pp. 1735-1755, 2012

[9] S. P. Singh, S. C. Sharma, "A survey on cluster based routing protocols for wireless sensor networks", Procedia Computer Science, Vol. 45, pp. $687-695,2015$

[10] W. K. Lai, C. S. Fan, L. Y. Lin, "Arranging cluster sizes and transmission ranges for wireless sensor networks", Information Sciences, Vol. 183, No.1, pp. 117-131, 2012

[11] W. R. Heinzelman, A. Chandrakasan, H. Balakrishnan, "Energyefficient communication protocol for wireless microsensor networks", IEEE 33rd Hawaii International Conference on System Sciences, Hawaii, USA, pp. 1-10, 2000

[12] W. B. Heinzelman, A. P. Chandrakasan, H. Balakrishnan, "Application specific protocol architecture for wireless microsensor networks", IEEE Transactions on Wireless Networking, Vol. 1, No. 4, pp. 660-670, 2002

[13] S. Lindsey, C. Raghavendra, K. M. Sivalingam, "Data gathering algorithms in sensor networks using energy metrics", IEEE Transactions on Parallel and Distributed System, Vol. 13, No. 9, pp. 924-935, 2002

[14] O. Younis, S. Fahmy, "HEED: A hybrid, energy-efficient, distributed clustering approach for ad hoc sensor networks", IEEE Transactions on Mobile Computing, Vol. 3, No. 4, pp. 366-378, 2004

[15] M. Xiaoyan, Study and design on clustering routing protocols for wireless sensor network, Ph.D Dissertation, Zhejiang University, Hangzhou, China, 2006

[16] X. Fang, Y. Song, "Improvement on LEACH protocol of wireless sensor network (E-LEACH)", International Conference on Sensor Technologies and Applications, Valencia, Spain, 2007

[17] M. B. Yassein, A. Alzou, Y. Khamayseh, W. Mardini, "Improvement on LEACH protocol of wireless sensor network (VLEACH)", International Journal of Digital Content: Technology and its Applications, Vol. 3, No. 2, pp. 132-136, 2009

[18] G. Ran, H. Zhang, S. Gong, "Improving on LEACH protocol of wireless sensor network using fuzzy logic", Journal of Information Science, Vol 7, No. 3, pp. 767-775, 2010

[19] N. M. A. Latiff, C. C. Tsimenidis, B. S. Sharif, "Energy aware clustering for wireless sensor networks using particle swarm optimization", $18^{\text {th }}$ Annual IEEE International symposium on Personal, Indoor, and Mobile Radio Communications, Athence, Greece, 2007

[20] J. Tillet, R. Rao, F. Sahin, "Cluster head identification in ad-hoc sensor networks using particle swarm optimization", IEEE International Conference on Personal Wireless Communications, New Delhi, India, pp. 201-205, 2002

[21] M. Azharuddin, P. K. Jana, "Particle swarm optimization for maximizing lifetime of wireless sensor network", Computers and Electrical Engineering, Vol. 51, pp. 26-42, 2016

[22] S. Chelbi, M. Abdouli, M. Kaddes, C. Duvallet, R., Bouaziz, "An unequal cluster based routing protocol based on data controlling for 
wireless sensor network", International Journal of Electrical and Computer Engineering, Vol. 6, No. 5, pp. 2403-2414, 2016

[23] J. Kennedy, R. C. Eberhart, "Particle swarm optimization", IEEE International Conference on Neural Networks, Piscataway, NJ, USA, pp 1942-1948, 1995

[24] R. V. Kulkarni, G. K. Venayagamoorthy, "Particle swarm optimization in wireless sensor network: a brief survey", IEEE Transactions on System, Man, and Cybernetics-Part C: Applications and Reviews, Vol. 41, No. 2, pp. 262-267, 2011

[25] K. Khan, W. Goodridge, "Energy aware Ad-Hoc on demand multipath distance vector routing", International Journal of Intelligent Systems and Applications, Vol. 7, No. 7, pp. 50-56, 2015

[26] http://file.scirp.org/Html/41-7601045_70085.htm

\section{AUTHORS PROFILE}

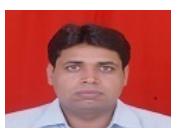

Santar Pal Singh is a Ph.D. student in Computer Engineering discipline, DPT, Indian Institute of Technology Roorkee (India) since July 2013.

He received the B.Tech in Computer Sc. \& Engineering from Kamla Nehru Institute of Technology, Sultanpur (U.P.) in 2001 and the M.Tech in Computer Sc. \& Engineering from Samrat Ashok Technological Institute, Vidisha (M.P.) in 2006. He has more than 10 years of teaching and research in area of computer science and engineering His present research interest includes Ad-hoc and wireless sensor networks, network security.

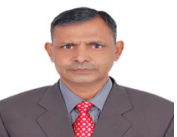

Professor Subhash C. Sharma received the M.Sc. (Electronics), M.Tech. (Electronics \& Communication Engg.) and Ph.D. (Electronics \& Computer Engg.) from IIT Roorkee (erstwhile University of Roorkee)

He has published over two hundred research papers in national and international journals/conferences and supervised more than 30 projects/dissertation of PG students. He has supervised $14 \mathrm{PhDs}$ in the area of Computer Networking, Wireless Network, Computer Communication and continuing supervising Ph.D. students in the same area. He has successfully completed several major research projects independently funded by various Govt. Agencies like AICTE, CSIR, MHRD, DST, and DRDO. 